\title{
Intraneural Venous Malformations of the Median Nerve
}

\author{
Sara Alicia González Porto, Alba González Rodríguez, José Midón Míguez \\ Department of Plastic Surgery, Hospital Universitario de A Coruña, A Coruña, Spain
}

\begin{abstract}
Venous malformations arising from the peripheral nerve are a rare type of vascular malformation. We present the first case of an intraneural venous malformation of the median nerve to be reported in a child and review the previous two cases of median nerve compression due to a venous malformation that have been reported. These cases presented with painless masses in the volar aspect of the wrist or with symptoms suggestive of carpal tunnel syndrome. Clinical suspicion should lead to the use of Doppler ultrasonography as the first-line diagnostic tool. Magnetic resonance imaging and histopathology can confirm the diagnosis, as phleboliths are pathognomonic of venous malformations. Surgical treatment appears to be the only modality capable of successfully controlling the growth of an intraneural malformation. Sclerotherapy and radiotherapy have never been used to treat this type of malformation.
\end{abstract}

Keywords: Hemangioma / Median nerve / Vascular malformation / Carpal tunnel syndrome

\author{
Correspondence: \\ Sara Alicia González Porto \\ Department of Plastic Surgery, \\ Hospital Universitario de A Coruña, \\ Xubias de Arriba 84, 15006 A Coruña, \\ Spain \\ Tel: +34-63-531-4161 \\ Fax: +34-98-650-3734 \\ E-mail: sarali.gonzalezporto@gmail. \\ com
}

We would like to thank Marta Gago Pesado for reviewing the translation of the manuscript, and Rose Hernández Cancela for providing us with the histopathological images.

No potential conflict of interest relevant to this article was reported.

Received: 19 Dec $2015 \bullet$ Revised: 10 Mar $2016 \bullet$ Accepted: 27 Apr 2016

pISSN: 2234-6163・ elSSN: 2234-6171・http://dx.doi.org/10.5999/aps.2016.43.4.371 • Arch Plast Surg 2016;43:371-373

\section{INTRODUCTION}

Venous malformations arising in peripheral nerves are a rare type of vascular malformation, and rarely origínate from the vasa nervorum. Only one case of intraneural venous malformation of the median nerve has been reported in the literature [1]. A case reported by Muchemwa et al. [2] involved an intramuscular venous malformation that led to extrinsic compression of the median nerve. Both cases presented with the symptoms of carpal tunnel syndrome. We present the first case of an intraneural venous malformation of the median nerve to be reported in a child.

\section{CASE REPORT}

A two-year-old male presented to our department with a palpable asymptomatic mass in his right palm that was present at birth and had subsequently exhibited a slow and steady pattern of growth (Fig. 1). Imaging showed the typical features of venous malformations [3]. Doppler ultrasonography indicated the presence of an irregularly shaped space-occupying lesion with multiple chambers and a low level of blood flow between the flexor retinaculum and the external flexor tendons, next to the branch of the nerve innervating the thumb and index finger, and magnetic resonance imaging (MRI) showed a heterogeneous signal, with hyperintensity on T2-weighted imaging and after

Copyright (C) 2016 The Korean Society of Plastic and Reconstructive Surgeons

This is an Open Access article distributed under the terms of the Creative Commons Attribution Non-Commercial License (http://creativecommons.org/

licenses/by-nc/4.0/) which permits unrestricted non-commercial use, distribution, and reproduction in any medium, provided the original work is properly cited. I www.e-aps.org 


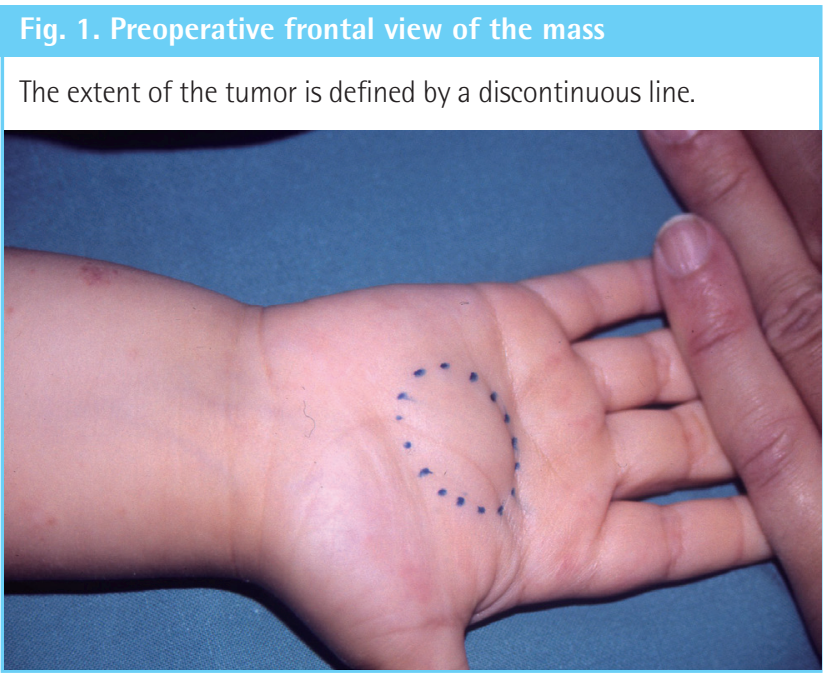

\section{Fig. 2. Intraoperative view}

The surgical approach consisted of a longitudinal incision along the palm of the right hand, which revealed a purple mass that was carefully dissected from the superficial tendinous fascicles. Interfascicular microdissection of the median nerve required optic magnification and was performed under limb ischemia.

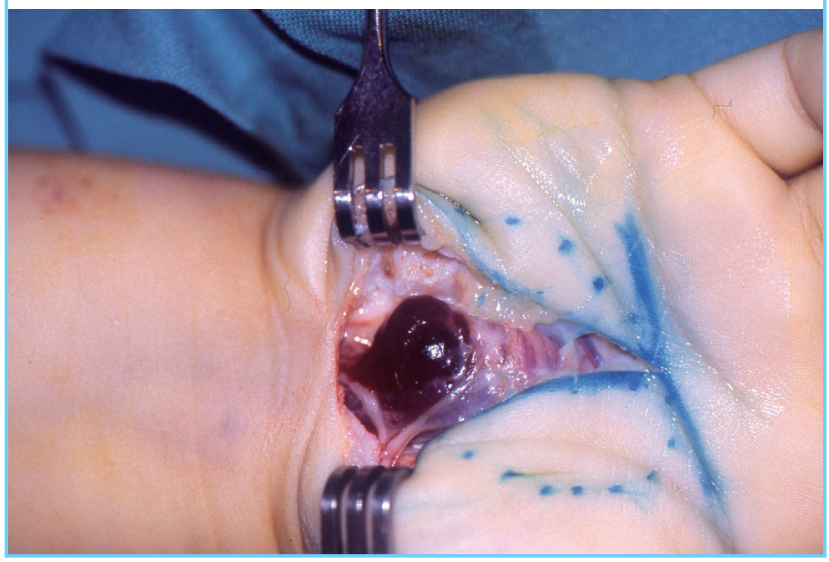

gadolinium administration, and hypointensity on T1-weighted imaging.

It was decided to excise the mass in order to verify the diagnosis of a vascular malformation, and to control the growth of the tumor. Surgery showed a purple mass that was $2.5 \mathrm{~cm}$ long (Fig. 2 ), and interfascicular microdissection of the median nerve was required; nevertheless, neither nerve grafts nor sacrificing any fascicle was necessary. Despite the performance of internal neurolysis, neither sensory nor motor symptoms were observed in the postoperative period.

Histopathological analysis revealed ectatic channels, surrounded by a thin layer of endothelial cells without hyperplasia (Fig. 3). Immunostaining with GLUT-1, WT-1 and D2-40 was negative. These results were compatible with a venous malformation. A year after the intervention, the patient presented to our de-

\section{Fig. 3. Cross section of the mass \\ Microscopic histopathology demonstrated the presence of multiple ectatic channels, surrounded by a thin layer of endothelial cells with- out hyperplasia $\left(H \& E_{1} \times 400\right)$.}

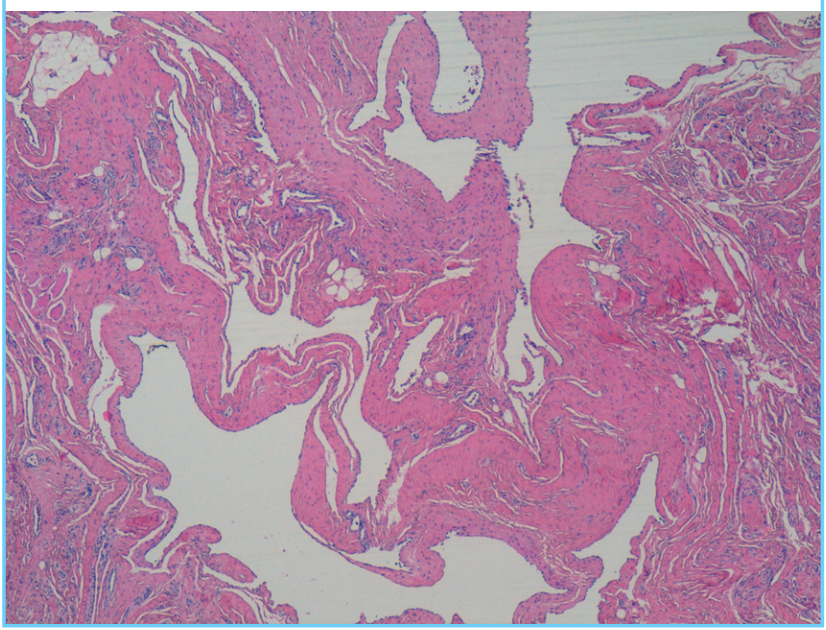

Fig. 4. Reintervention intraoperative view

Intraoperative view of the second operation showing a bluish irregular mass, measuring $7 \times 2.5 \times 1.5 \mathrm{~cm}$, which surrounded and infiltrated the median nerve, affecting the interfascicular space from the distal third of the forearm to the distal palmar crease, above the metacarpophalangeal joints.

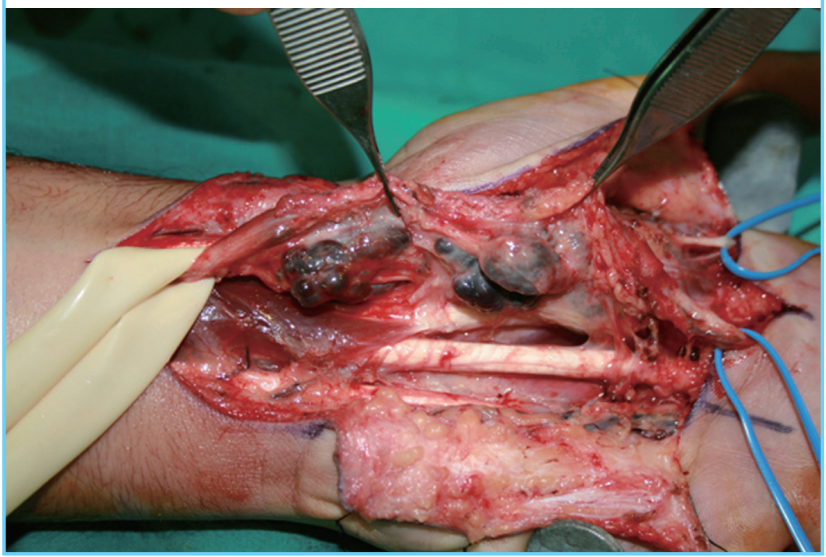

partment with a local relapse. No sensory, motor, or electromyographic deficiencies were found. The mass continued to grow steadily and after 10 years of follow-up, the patient reported local pain and numbness in the thumb, index, middle and ring fingers, and a new operation was planned.

The reintervention revealed a bluish irregular mass (Fig. 4), that was $7 \mathrm{~cm}$ long and surrounded and infiltrated the median nerve. The histopathology results were the same as those obtained after the first operation; however, this time phleboliths were found (Fig. 5), confirming the diagnosis of venous malformation. Phleboliths and calcifications, pathognomonic of venous malformations, can be found after episodes of local intra- 


\section{Fig. 5. Surgical specimen in the reintervention}

Surgical specimen from the second operation, consisting of the venous malformation and four phleboliths, pathognomonic of a venous malformation.

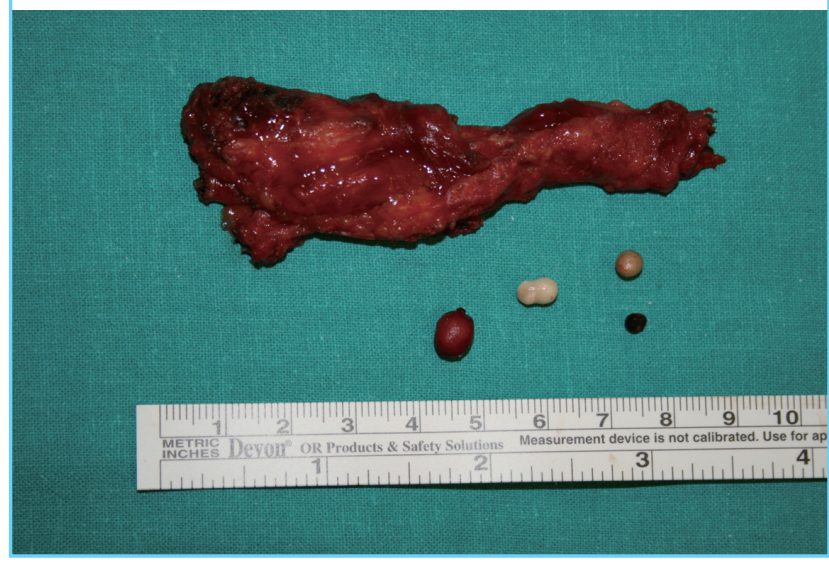

vascular coagulation, if the venous malformations are medium to large in size [3]. This may have been the reason why they appeared in the surgical specimen from the second intervention $(7.5 \mathrm{~cm}$ in length), but not in the surgical specimen from the first operation $(2.5 \mathrm{~cm})$.

Although macroscopic complete excision seemed to be achieved, a new local relapse was clinically detected and confirmed by MRI 12 months subsequently (Fig. 6).

\section{DISCUSSION}

The standard of care for intraneural venous malformations requires a multidisciplinary approach and a thorough assessment of the location and extension of the lesion.

Sclerotherapy, laser therapy (neodymium), cryotherapy and pressure therapy are possible alternatives for venous malformations affecting other structures, but have never been used to treat intraneural malformations.

With regard to intraneural venous malformations, surgery is the only treatment modality capable of controlling growth and compressive symptoms, although it was a temporary solution in the case presented in this report due to relapse after 12 months after the first and second operations [4]. In the case reported by Hariri et al. [1] no relapse took place over 12 months of follow-

\section{Fig. 6. Magnetic resonance imaging of the relapse}

Of particular note is the hyper-intense signal of the mass on the T2-weighted sequence, with the mass located between the flexor retinaculum and the external flexor tendons in the wrist and extending from the distal third of the forearm to the distal palmar crease.

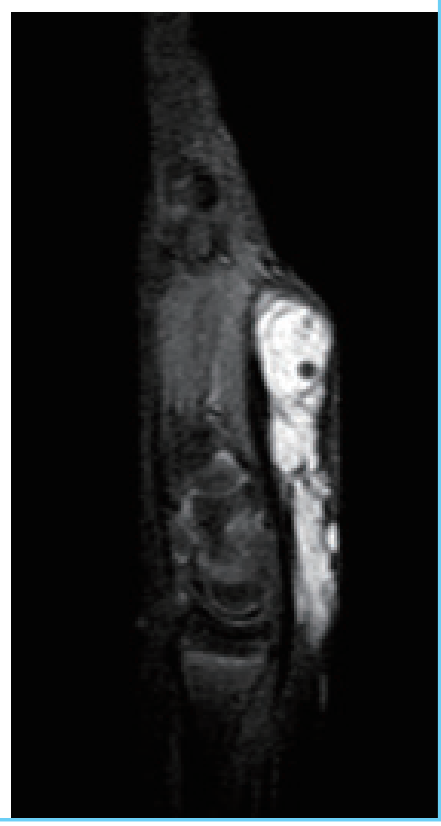

up. It is possible to avoid potential surgical difficulties, such as the tendency towards bleeding due to the vascular richness of the malformed tissue, by employing meticulous surgical techniques, using optical magnification and limb ischemia. Postoperative complications did not occur in any of the three cases.

\section{REFERENCES}

1. Hariri A, Cohen G, Masmejean EH. Venous malformation involving median nerve causing acute carpal tunnel syndrome. J Hand Surg Eur Vol 2011;36:431-2.

2. Muchemwa FC, Ishihara T, Matsushita S. Intramuscular venous malformation in the upper arm with gross calcifications and compression of the ulnar nerve. Scand J Plast Reconstr Surg Hand Surg 2007;41:93-5.

3. Colletti G, Valassina D, Bertossi D, et al. Contemporary management of vascular malformations. J Oral Maxillofac Surg 2014;72:510-28.

4. Cox JA, Bartlett E, Lee EI. Vascular malformations: a review. Semin Plast Surg 2014;28:58-63. 